\title{
Ventricular myoarchitecture in tetralogy of Fallot
}

\author{
Damian Sanchez-Quintana, Robert H Anderson, Siew Yen Ho
}

\begin{abstract}
Background-Little attention has been paid to the architecture of the muscle fibres of the ventricular walls in congenitally malformed hearts. In this study the gross pattern of myocardial fibres in normal hearts was compared with that in cases of tetralogy of Fallot.

Methods and results-After morphological examination nine specimens with tetralogy were dissected to study the ventricular myoarchitecture. Changes were found in the shape of the malformed ventricles. The ventricular walls were arranged in layers in all hearts. Superficial and deep layers were present in both ventricles, with the superficial layer showing a more oblique orientation in the specimens with tetralogy than in normal hearts. Modifications of muscle fibre that were related to the type of malformation were seen in the deep layer. A middle layer was present in the left ventricles of normal hearts and specimens with tetralogy: this showed a horizontal orientation in both groups. In contrast, a middle layer was found in the right ventricle only in specimens showing tetralogy.

Conclusions-The malformed hearts showed modifications in ventricular shape, in the arrangement of muscle in the right ventricle, and in the overall myoarchitecture. These changes could well be the consequence of the same agent (or agents) that caused the structural defect.
\end{abstract}

(Heart 1996;76:280-286)

Departamento de

Anatomia Humana,

Facultad de Medicina,

Universidad de

Extremadura,

Badajoz, Spain

D Sanchez-Quintana

Department of

Paediatrics, National

Heart and Lung

Institute, Dovehouse

Street, London

R H Anderson

$S$ Y Ho

Correspondence to:

Dr S Y Ho, Department of

Paediatrics, National Heart

and Lung Institute,

Dovehouse Street, London SW3 6LY.

Accepted for publication

25 April 1996
Keywords: anatomy; tetralogy of Fallot; ventricular myoarchitecture

That the heart is a muscular organ with a complex architectural arrangement of its ventricular fibres was known to Harvey ${ }^{1}$ over 300 years ago. Many workers since then have investigated this architecture in the normal heart, mostly basing their findings on gross dissection. ${ }^{2-8}$ Others have combined dissection and histology. ${ }^{910}$ More recently, sophisticated methods have been used based on threedimensional reconstruction from serial sections. ${ }^{112}$ Comparison of these various studies has revealed several controversies. Some argue that there are discrete systems of fibres in each ventricle, ${ }^{210}$ whereas others describe a single system encompassing both ventricles. ${ }^{8}$ Some have proposed the fibrous trigones as points of anchorage of the muscle fibres, ${ }^{45}$ while others have proposed the aortic and pulmonary roots as the only areas of insertion. ${ }^{8}$ Yet another view is that the myocardial fibres branch from one another, as if they were inserted in adjacent muscle fibres, rather than taking their origin or insertion from the fibrous skeleton. ${ }^{9}$ Despite these discrepancies, these studies have shown that the myocardium represents a complex three-dimensional network of cells, with each layer having a preferred orientation. It seems reasonable to propose that changes in heart shape, and in the force generated during systole, depend on the relative orientation of, and interaction between, these bundles of fibres. Studies of the muscular architecture, for the most part, have been confined to normal hearts, with few studies comparing dissections of normal and congenitally malformed hearts. ${ }^{13}$ To extend this field of study we compared the ventricular myoarchitecture of the normal heart with that in one congenital malformation, namely tetralogy of Fallot.

\section{Methods}

Nine specimens from cases of tetralogy of Fallot and four normal hearts were studied. The specimens with tetralogy of Fallot were selected from the cardiopathological collection of the National Heart and Lung Institute, Royal Brompton Hospital, London. To be regarded as an example of tetralogy, a heart had to show four features: namely a ventricular septal defect, subpulmonary stenosis or atresia, biventricular origin of the leaflets of the aortic valve, and right ventricular hypertrophy. The patients with tetralogy of Fallot from whom the hearts were obtained ranged in age from eight hours to 59 years. The normal hearts came from subjects aged from seven to 61 years old who died of causes unrelated to cardiac pathology. The normal hearts were fixed by immersion in $10 \%$ formaldehyde for about two weeks before study. The hearts from the cardiopathological collection have been fixed in $10 \%$ formaldehyde for many years, but their initial preparations were similar. They were initially fixed intact by perfusion through the orifice of the inferior caval vein after ligation of other connecting vessels.

Our specimens formed three subgroups representing the range of morphologies in tetralogy of Fallot. One specimen showed rudimentary formation of the pulmonary valve (so-called absent pulmonary valve syndrome). 
Valvar and subpulmonary infundibular stenosis was found in six specimens. Pulmonary atresia was present in the remaining two.

The protocol used for dissection was similar to that followed in earlier studies, ${ }^{14}$ but in some cases the atria were not detached along the line of the atrioventricular grooves. The hearts were dissected with watchmakers' tweezers and scissors, taking care not to disrupt superficial and deep myocardial fibres when the epicardium or endocardium was removed. Special care was taken to study the anatomy of the fibrous skeleton, along with the interrelations between myocardial fibres and the leaflets of the arterial atrioventricular valves. In studying architecture of the myocardium, we used the term "muscle fibre" to describe a bundle of myocytes visible by gross inspection. Thus fibres aligned in the same orientation were exposed by step-by-step peeling from the subepicardium to the subendocardium. Each step was documented by serial photography. The myocardial layers were described as superficial, deep, and middle; with each layer being distinguished on the basis of a change in orientation of muscular fibres from the adjacent layer.

\section{Results}

EXTERNAL MORPHOLOGICAL FEATURES

In normal hearts the aortic orifice occupies a key position between the atrioventricular orifices and that of the pulmonary valve. The right edge of the ventricular mass is sharp (acute) when seen from the apex, in contrast to the left margin which is much more rounded (obtuse margin) (fig 1A). Vortices are seen on the tips of both ventricles, although the left vortex is always sharper and forms the apex of the heart itself (fig 1A).
These typical external morphological features of the normal hearts were altered in the malformed hearts. In our first case, characterised by rudimentary formation of the pulmonary valve, the anterior wall of the right ventricle in the outflow tract was dilated, while the aortic root was displaced markedly rightwards (figs $1 \mathrm{~B}$ and $2 \mathrm{C}$ ). Right ventricular hypertrophy was obvious, and both margins of the ventricular mass were obtuse. Moreover, at the level of the apex, the right vortex was a similar size to the left (fig 1B). The interventricular grooves were clearly evident.

When there was pulmonary infundibular stenosis together with valvar stenosis, right ventricular hypertrophy remained a significant feature (fig 1C). A bifid apex was formed by both ventricles, but the right margin remained sharp. Such morphological features were observed irrespective of the nature of pulmonary valve stenosis.

When tetralogy was complicated by pulmonary atresia, right ventricular hypertrophy was particularly marked, enlarging the anteroposterior diameter of the heart and giving obtuse margins on both right and left sides (fig 1D). Although, when seen from the front or posteriorly, the apex seemed to be formed by both ventricles (figs $2 E$ and $F$ ), when viewed end-on it was shown to be formed only by the vortex of the left ventricle (fig 1D).

\section{MYOARCHITECTURE}

In all the hearts it was possible to distinguish three different layers of muscle fibres: superficial (subepicardial), deep (subendocardial), and middle. The distinction between one layer and the next was made on the basis of a change in the orientation of the muscle fibres. No discrete planes or fibrous septa were pre-
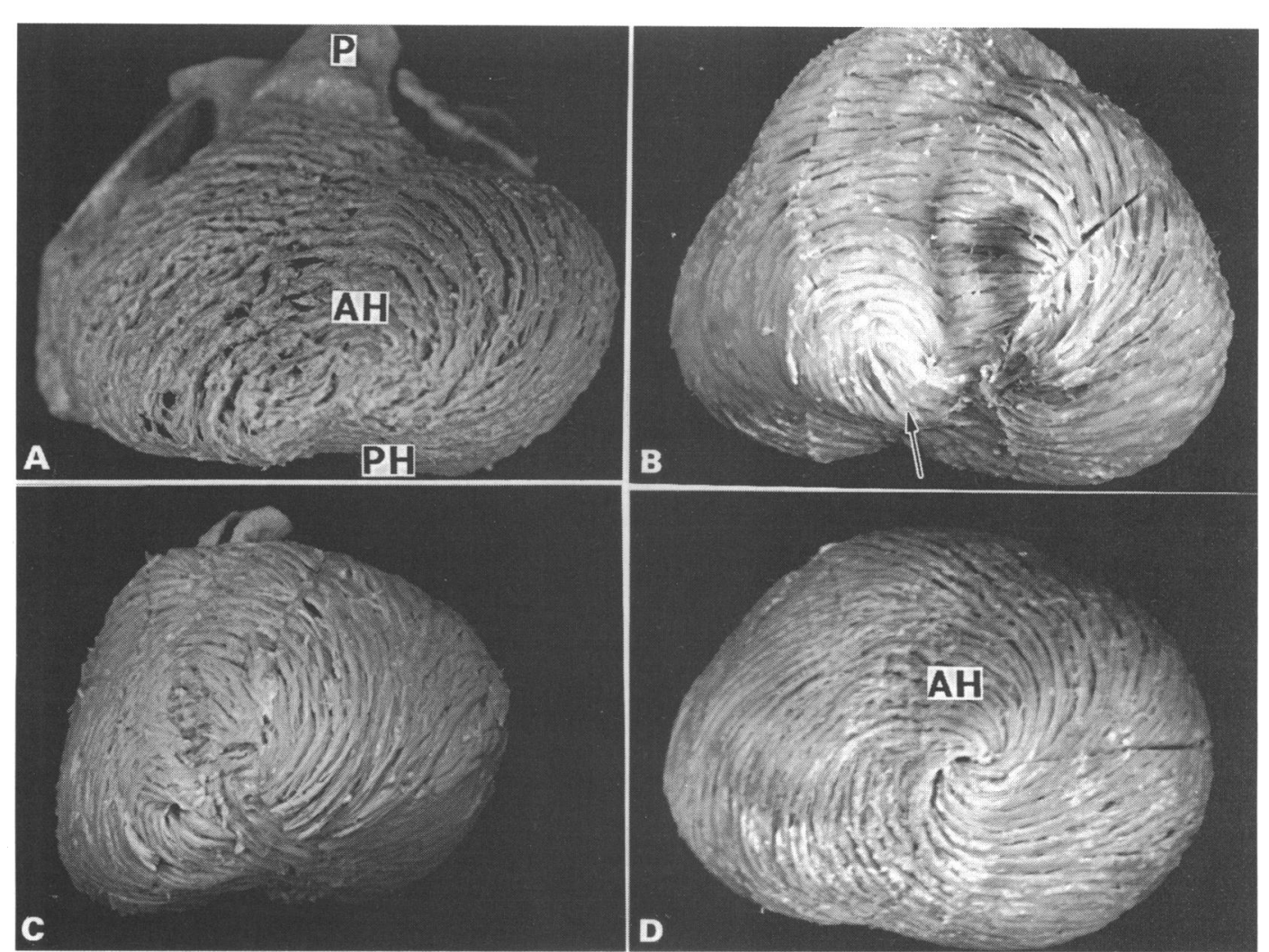

Figure 1 Apical views of the hearts of normal $(A)$ and tetralogy $(B, C, D)$
specimens, showing the arrangement of the superficial fibres at the apex. In $(A)$ note the anterior $(A H)$ and posterior (PH) horns at the level of the left vortex. Note the hypertrophy of the right ventricle and the marked separation of the vortices in the case with absence of the leaflets of the pulmonary valve (B) and subpulmonary infundibular stenosis $(C)$, with some fibres of the diaphragmatic aspect (arrow) of the heart ending in the right vortex. In pulmonary atresia (D) only the left vortex was observed, showing an increase of fibres at its anterior horn $(A H)$, with both margins of the heart being very obtuse. $P$, pulmonary trunk. 


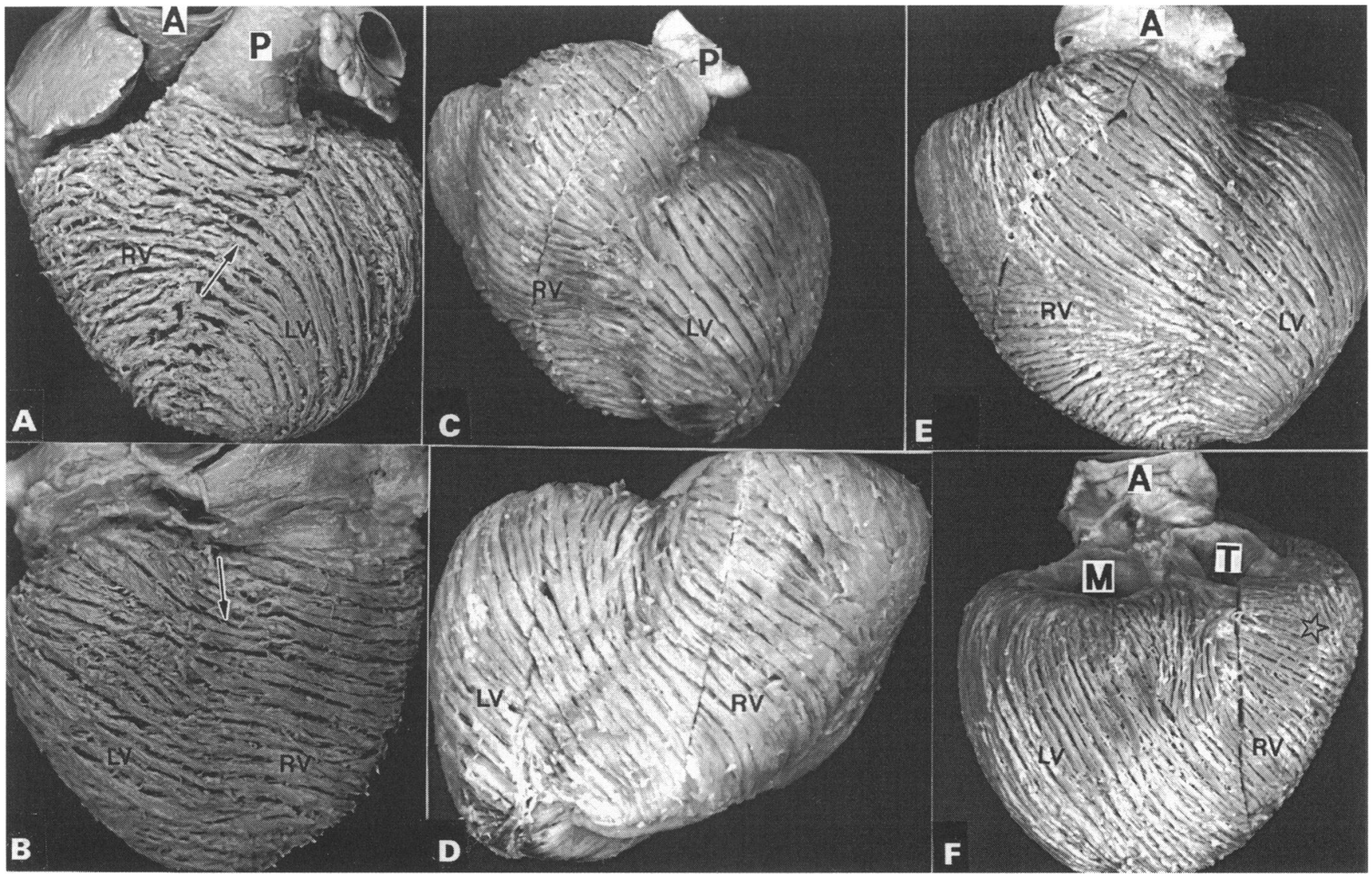

Figure 2 Front and back views of the hearts of normal $(A$ and $B$ ) and tetralogy (C-F) specimens, showing the arrangement of the superficial layer. In $A$ and $B$ the fibres run obliquely across the interventricular grooves (arrow). Note the right ventricular hypertrophy in the case with rudimentary formation of the pulmonary valve (C and D), and pulmonary atresia $(E$ and $F)$, with fibres more obliquely or longitudinally orientated in the right ventricle of specimens with tetralogy. A, aorta; $P$, pulmonary trunk; $T$ and $M$, tricuspid and mitral valvar orifices; star; longitudinal and transverse fibres.

sent between the layers. Obvious differences were noted, however, in the arrangement and orientation of the layers in the ventricular walls, both between right and left ventricles and between the normal and malformed hearts.

\section{Superficial layer}

The architecture of the superficial layer showed features common for both normal hearts and those with tetralogy. This layer was traced from the base of the heart toward the apex, extending from one ventricle to the other (fig 2). At the level of the apex the superficial layer invaginated in a spiral pattern to form a deep or subendocardial layer for each ventricle. The invagination at the left vortex formed anterior and posterior horns. The anterior horn was derived from muscle fibres arising from the posterior region of the base of the heart, whereas the posterior horn was formed by fibres arising from the anterior region of the base (fig 1). The fibres on the sternocostal aspect ran obliquely from right to left, crossing the anterior interventricular groove (fig $2 \mathrm{~A}, \mathrm{C}$, and $\mathrm{E}$ ), while on the diaphragmatic aspect they ran left to right and crossed the posterior interventricular groove (fig $2 \mathrm{~B}, \mathrm{D}$, and $\mathrm{F}$ ). In normal hearts, the fibres in the superficial layer ran more horizontally in the right than in the left ventricle (figs $2 \mathrm{~A}$ and B).

Markedly different features were seen in hearts with tetralogy. At the base, the speci- men with rudimentary formation of the leaflets of the pulmonary valve showed a more oblique orientation of the fibres distally on the dilated subpulmonary outflow. In contrast, in the specimens with pulmonary atresia, the fibres showed an almost circular arrangement around the aorta. An intermediate configuration was found in the cases with pulmonary valvar and infundibular stenosis.

The specimen with rudimentary leaflets of the pulmonary valve and those with pulmonary valve stenosis showed a marked separation between the ventricular vortices at the apex, with major development of the right vortex, composed of muscle fibres arising directly from the posterior region of the base (figs $1 \mathrm{~B}$ and $\mathrm{C}$ ). In contrast, in pulmonary atresia, the fibres formed a spiral pattern only around the left ventricular vortex, with an increase in the fibres of the anterior horn (fig 1D).

The sternocostal right ventricular fibres were more obliquely orientated than for the normal heart, particularly in the hearts with pulmonary atresia (fig $2 \mathrm{E}$ ). At the diaphragmatic aspect, the left ventricular fibres ran in a more oblique orientation, again markedly so in pulmonary atresia, where they acquired an almost longitudinal orientation (fig $2 \mathrm{D}$ and $\mathrm{F}$ ). In all the hearts, the fibres were irregularly orientated in the upper third of the diaphragmatic surface of the right ventricle, next to the right margin, with longitudinal fibres intermingling with transverse ones (fig $2 \mathrm{~F}$ ). 
Deep layer

In the normal hearts the deep or subendocardial layer comprised preferentially arranged longitudinal fibres which passed through the vortices and coursed in three different directions, toward the papillary muscles, to the atrioventricular and arterial orifices, and to the membranous interventricular septum.

Multiple differences were seen in the hearts with tetralogy. In the case with absent or rudimentary pulmonary leaflets or those with pulmonary valvar stenosis, the fibres from the right vortex ran upward longitudinally to follow the septomarginal trabeculation and the ventriculo-infundibular fold to the tricuspid annulus (fig 3A). In all cases, as the fibres approached the site of the ventricular septal defect, they divided. Some fibres bordered the defect anteriorly, following the outlet septum, while others extended posteriorly, following the junction of the ventriculo-infundibular fold with the septomarginal trabeculation (fig 3B-D). The fibres above the outlet septum ran upwards, reaching the pulmonary valve to form part of the subpulmonary infundibulum (fig 3B and C). The fibres surrounding the ventricular septal defect were closely related to the leaflets of the aortic valve. In those with pulmonary infundibular stenosis, the septoparietal trabeculations were formed by a thick bundle of fibres arranged longitudinally, which reached the pulmonary valve superiorly to narrow the subpulmonary outflow tract.

In the cases with pulmonary atresia the outlet septum was either rudimentary or absent, with the aorta being the only arterial outlet. The subendocardial fibres ascended longitudinally in the right ventricle (fig 3D). Toward the rim of the ventricular septal defect some fibres extended anteriorly and ran towards the
Figure 3 Deep layer of the right ventricle $(A, B$, $C$, D) and left ventricle $(E, F)$ from specimens with tetralogy that have been sectioned and opened longitudinally. Note that in the case with

rudimentary leaflets of the pulmonary valve $(A)$, the longitudinally orientated fibres in the septomarginal trabeculation continue as transverse fibres in the outlet septum, and that in subpulmonary infundibular stenosis (B) hypertrophy of septoparietal trabeculations narrows the pulmonary outflow tract (arrows). The fibres that border the ventricular septal defect and the overriding aortic valve $(C)$ run toward the aortic valve. In the case of pulmonary atresia (D) the fibres near the rim of the ventricular septal defect divide to pass anteriorly and posteriorly to the defect. In the left ventricle $(E, F)$ the fibres divide near the rim to approach the aortic valve. $A$, aorta; asterisk, ventricular septal defect; $M L$, middle layer of the left ventricle; OS outlet septum; $P$

pulmonary trunk; ST septomarginal

trabeculation; $T$, tricuspid valvar orifice; $P M$, papillary muscle.
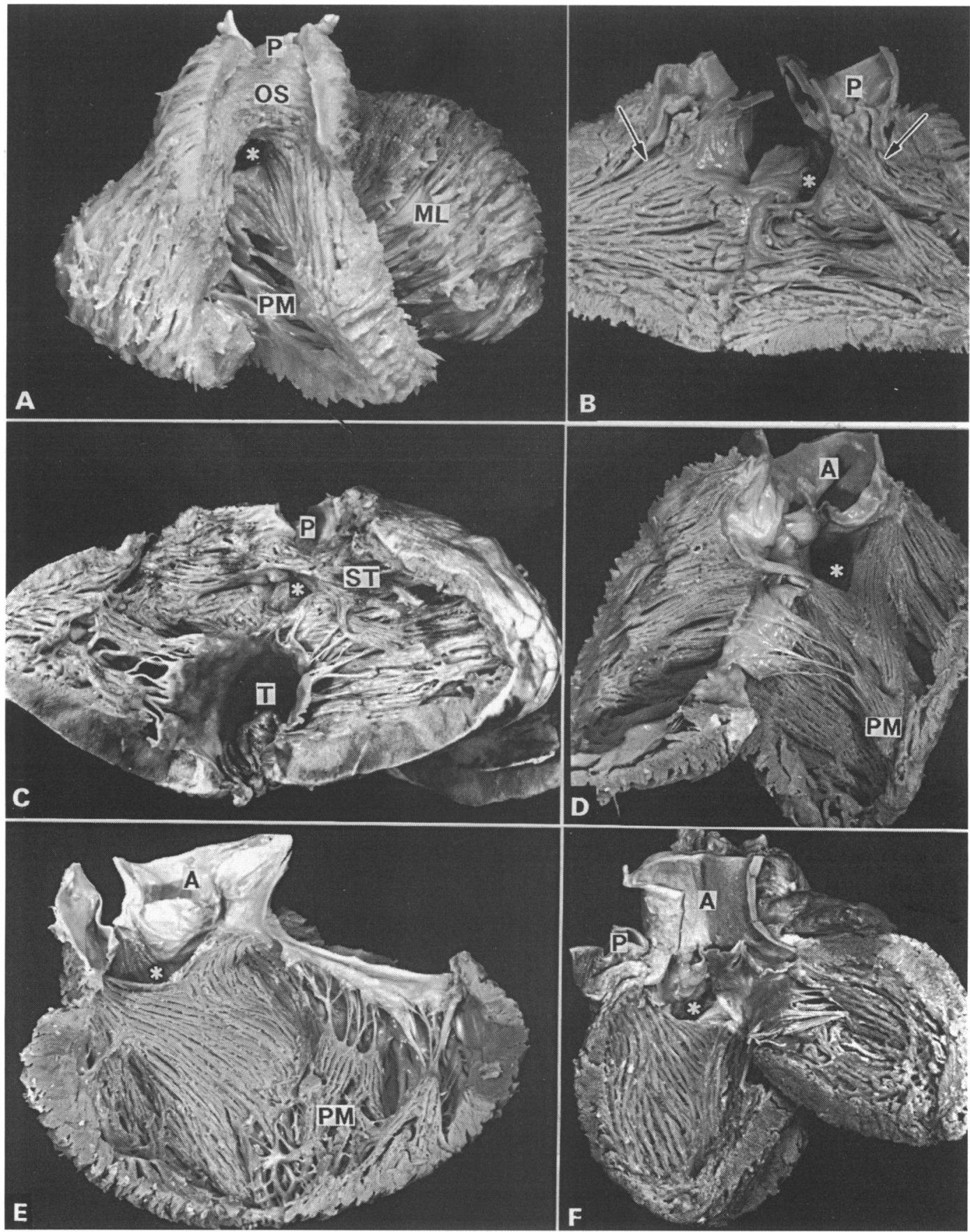
Figure 4 Front $(A)$ and apical (B) views of the normal heart showing a circular pattern of muscle fibres in the middle layer of the left ventricle. Front (C) and interventricular (D) views in a specimen with tetralogy (pulmonary atresia) show the middle layer together with hypertrophy of the right ventricle (C) but at the level of the ventricular septal defect (asterisk) there is discontinuity and only the deep layer (arrow) can be seen. $A$, aorta; $L V$, left ventricle; $R V$, right ventricle; $P$, pulmonary trunk.
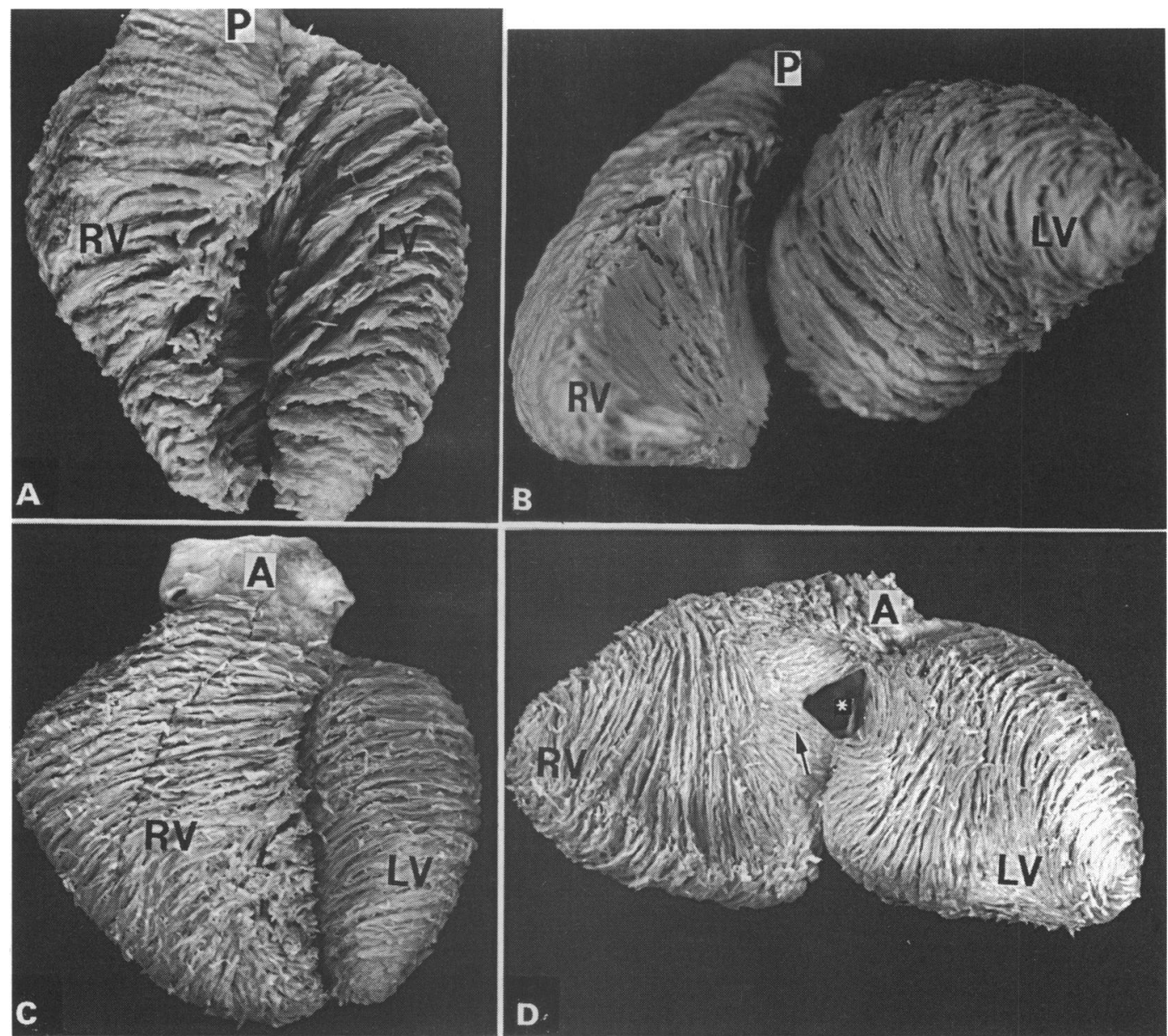

attachment of the right and left leaflets of the aortic valve. Others ran towards the non-coronary leaflet of the aorta (fig $3 \mathrm{D}$ and $\mathrm{E}$ ). A third, smaller, group of fibres crossed the rim of the defect and interwove with subendocardial fibres of the left ventricle (fig 3E).

The arrangement of the subendocardial fibres in the left ventricle was very similar in all hearts, approximating to the arrangement seen in the right ventricle in cases with pulmonary atresia. Other fibres of the left ventricle were arranged longitudinally, with insertions into the papillary muscles and the mitral annulus (fig 3F).

\section{Middle layer}

In normal hearts a thick middle layer of myocardial fibres was found only in the left ventricle, where it formed an almost circular pattern, with no apparent insertion into the aortic-mitral annulus. No proper middle layer was found in normal right ventricles, with no changes seen in the orientation of the fibres in the mid-portion of the ventricular wall (fig $4 \mathrm{~A}$ and $\mathrm{B}$ ).

Several changes were seen in the hearts with tetralogy. In all cases, the fibres of the left ventricle showed a marked circular arrangement (figs 4C and D). Most significantly, again in all types, the right ventricle possessed a welldefined middle layer composed of circular fibres. The layer was discontinuous only at the level of the ventricular septal defect, where the fibres of the subendocardial layer of the right ventricle could be seen (fig 4C and D).

\section{Discussion}

Our findings demonstrate a normal pattern of ventricular myoarchitecture characterised, in the left ventricle, by three concentric layers (superficial or subepicardial, middle, and deep or subendocardial). Only superficial and deep layers, in contrast, are found in the normal right ventricle. These findings are in keeping with several earlier descriptions, ${ }^{56910}$ but are in marked contrast to the hypothesis which proposes a single system of spiralling fibres extending across the ventricles from the aortic to the pulmonary root. ${ }^{8}$

Marked departures from this normal arrangement were found in all hearts studied with the anatomical features of the various morphologic subsets of tetralogy of Fallot. Despite some continuing disagreements regarding the interpretation and nomenclature of muscle bands surrounding the ventricular septal defect, ${ }^{15-17}$ and disagreements about the extent of subpulmonary infundibular stenosis, ${ }^{1618}$ it is generally agreed that the morphological hallmarks that unify tetralogy are a ventricular septal defect, subpulmonary infundibular stenosis, biventricular connection of the leaflets of the aortic valve, and hypertrophy of the right ventricle. The classic anatomical feature that is diagnostic in most hearts with tetralogy of Fallot is antero-cephalad deviation of the outlet septum, ${ }^{16}$ such that this septum takes up an exclusively right ventricular position. Despite their obvious similarities, nonetheless, no two cases of tetralogy are exactly alike, ${ }^{19}$ a feature of major surgical sig- 
nificance. These disparities were further confirmed by our dissections of the myocardial architecture. Despite the individual differences, we could divide our material into one specimen with absence of the leaflets of the pulmonary valve, and others with either pulmonary stenosis or with pulmonary atresia. In the first two subsets, the anatomical hallmark, the antero-cephalad deviation of the outlet septum, is readily apparent irrespective of the length of the subpulmonary infundibulum. The outlet septum was deviated in a similar fashion in one of the hearts with pulmonary atresia, but was absent in the other. It is arguable whether hearts with pulmonary atresia should be included in the overall category of tetralogy. Indeed, our specimen with absence of the outlet septum exhibits overriding of the aorta, pulmonary atresia at the ventriculo-arterial junction, and a doubly committed and juxta-arterial ventricular septal defect. But despite having pulmonary atresia, rather than stenosis, these two hearts are still more closely related to tetralogy than to other subsets of hearts with different segmental combinations which are also described as pulmonary atresia with ventricular septal defect. ${ }^{20}$

Taken together, these morphological types differ from the normal heart and among themselves not only in terms of ventricular shape, but also with regard to the architecture of the ventricular muscle fibres. In general, the ventricular shape was dominated by the state of the right muscular outlet. In the presence of rudimentary formation of the leaflets of the pulmonary valve, the outlet tract was dilated, with more obtuse angles of the margins than in the normal ventricles. In contrast, the cases with pulmonary stenosis show obvious hypertrophy of the right ventricle, but with various lengths of outlet tracts, albeit usually larger than normal. The ventricular shape is more angular than in the heart with rudimentary leaflets of the pulmonary valve. In our particular hearts with pulmonary atresia, the subpulmonary outlet tract of the right ventricle was markedly reduced in one, and lacking in the other. The margins of the ventricles showed the most obtuse angles. In all forms of tetralogy of Fallot, therefore there is an altered ventricular configuration.

It has been suggested that the shape of the heart reflects an adaptation to the needs of both systole and diastole, and that those changes in ventricular shape may in themselves produce cardiac dysfunction. ${ }^{21}$ In this respect, therefore, it may well be significant that the basic architecture of the myocardium in tetralogy is also very different from that in the normal heart. The most significant finding is a circular middle layer in the right ventricle. Previous studies in normal hearts have demonstrated the existence of such a middle layer in the left, but not in the right ventricle. ${ }^{5-710}$ In the hearts with tetralogy, the overall architecture is markedly influenced by the presence of right ventricular hypertrophy. Earlier studies have already reported that, when the right ventricle is hypertrophied ${ }^{132122}$ it exhibits an increase of fibres at the level of the ventricular septum and right ventricular outlet, and as a consequence the architecture of the ventricular septum shows a convexity towards the left. Haemodynamic, histological, and morphometric studies have previously revealed hypertrophic changes in both ventricles in tetralogy..$^{23-25}$ It could be that the right ventricular middle layer is acquired postnatally as an adaptive change to the ventricular load, but the finding of a middle layer in the specimen from our youngest patient, a neonate, and also in the oldest patient, who was 59, suggests that the myocardial architecture is a further characteristic of the abnormal cardiac development. Clinical studies have shown persistent depressed function of the right ventricle in some cases, even subsequent to surgical repair. ${ }^{26-28}$ In these, increased myocardial fibrosis has been proposed as an important factor in restricting myocardial compliance. ${ }^{26-29}$ The abnormal myoarchitecture must be considered a potential additional factor. It has been demonstrated that rearrangement in orientation of muscle fibres, and changes in interstitial connective tissue matrix, can influence both the electrical and mechanical function of the heart. ${ }^{3031}$ Our previous studies $^{32}$ showed that a middle layer was lacking from the right ventricle of normal hearts in human specimens ranging in age from fetuses of 13 weeks' gestation to adults aged 90 . We need now, therefore, to explore the clinical significance of these anatomical findings. Our bias is that in tetralogy the abnormal ventricular architecture already present at birth represents an adaptative change in formation of the myocardial fibres, but we do not yet know at which developmental stage the middle layer is formed.

\section{LIMITATIONS OF THE STUDY}

Good necropsy examples of Fallot's tetralogy, especially those from subjects who have not had previous surgical interventions, are becoming increasingly scarce. For this study, we selected specimens from our collection that were basically intact; this will further limit the numbers that will be available for study in the future. For this reason, we have included hearts which, at first glance, are grossly different in their morphology but, nonetheless, satisfy the accepted criteria for description as tetralogy of Fallot. We did not study many hearts, none the less, all show the same basic differences in from normal myoarchitecture. We cannot increase the numbers because such an approach would mean destroying all our specimens with tetralogy, leaving a wide gap in the teaching value of our collection. Rather, we hope our findings will generate interest in this method of investigation among other morphologists, especially in centres that have collections of necropsied hearts that were established before the early days of cardiac surgery.

It is also pertinent that we did not attempt to quantify the changes in orientation of the fibres. Our technique is based on gross dissection, which eventually leaves little of the specimen. For meaningful assessment of the 
angular changes, histological sections through the full thickness of the ventricular walls are preferable, ${ }^{9}$ and this also is a fruitful area for future study.

During this investigation D S-Q was supported by grant DGICYT (PR95-057) from the Spanish government. SYH and RHA are supported by the British Heart Foundation.

1 Harvey W. An anatomical disquisition on the motion of the heart and blood in animals (1628). In: Willis FA, Keys TE eds Cardiac Classics. London: Henry Kimpton. 1941:19-79.

2 Pettigrew JB. On the arrangement of the muscular fibres in the ventricles of the vertebrate heart, with physiological
remarks. Philos Trans $R$ Soc Lond 1865;154:445-500.

remarks. Philos Trans $R$ Soc Lond $1865 ; 154: 445-500$.
3 Krehl L. Kenntniss der Fullung und Entleerung des Herzens. Abhandl Math Phys KI Koniglichen Saechs Ges Wiss 1891;29:341-62.

4 Mall FP. On the muscular architecture of the ventricles of the human heart. Am ₹ Anat 1911;11:211-66.

5 Robb JS, Robb RC. The normal heart, anatomy and physiology of the structural units. Am Heart $f 1942 ; 23$ : 455-67.

6 Grant PP. Notes on the muscular architecture of the left ventricle. Circulation 1965;32:301-8.

7 Streeter DD. Gross morphology and fiber geometry of the heart. In: Berne RM, Sperelakis N, Geiger SR, eds Handbook of physiology, the cardiovascular system. Baltimore: Williams and Wilkins. 1979:61-112.

8 Torrent-Guasp F. La estructura macroscopica del miocardio ventricular. Rev Esp Cardiol 1980;33:265-87.

9 Greenbaum RA, Ho SY, Gibson DG, Becker AF, Anderson RH. Left ventricular fibre architecture in man. Br Heart f 1981;54:248-63.

10 Fernandez-Teran MA, Hurle JM. Myocardial fiber architecture of the human heart ventricles. Anat Rec 1982; 204:137-47

11 McLean MR, Prothero J Coordinated three-dimensiona reconstruction from serial sections at macroscopic and serial sections at macros heart. Rec 1987;219:434-9.

12 Usson Y, Parazza F, Jouk PS, Michalowicz G. Method for the study of the three-dimensional orientation of the the study of the three-dimensional orientation of the nuclei of myocardial cells in fetal human heart by means of confocal scan

13 Becker AE, Caruso G. Congenital heart disease: a morphologist's view on myocardial dysfunction. In: Becker $\mathrm{AE}$, Losekoot TG, Marcelletti C, Anderson RH. eds.
Paediatric cardiology. Vol 3. London: Churchill LivingPaediatric cardiology.

14 Sanchez-Quintana D, Hurle JM. Ventricular myocardial architecture in marine fishes. Anat $\operatorname{Rec} 1987 ; 217$ 263-73.

15 Rosenquist GC, Sweeney LJ, Stemple DR, Christianson $\mathrm{SD}$, Rowe RD. Ventricular septal defect in tetralogy of Fallot. Am f Cardiol 1973;31:749-54.
16 Becker AE, Connor M, Anderson RH. Tetralogy of Fallot: a morphometric and geometric study. Am $\mathcal{f}$ Cardiol 1975;35:402-12.

17 Anderson RH, Becker AE, Van Mierop LHS. What should we call the "crista"?. Br Heart $\mathcal{F}$ 1977;39:856-9.

18 Van Praagh R, Van Praagh S, Nebesar RA, Muster AJ, Shina SN, Paul MH. Tetralogy of Fallot. Underdevelopment of the pulmonary infundibulum and its sequelae. Am $\mathcal{f}$ Cardiol 1970;26:25-33.

19 Lev M, Eckner FAO. The pathologic anatomy of tetralogy of Fallot and its variations. Dis Chest 1964;45:251-61.

20 Anderson RH, Devine W, del Nido P. The surgical anatomy of tetralogy of Fallot with pulmonary atresia rather than pulmonary stenosis. I Card Surg 1991;6: 41-59.

21 Hutchins GM, Bulkley BH, Moore GW, Piasio MA, Lohr FT. Shape of the human cardiac ventricles. $A m \mathcal{F}$ Cardiol 1978;41:646-54.

22 Sanchez-Quintana D, Garcia-Martinez V, Hurle JM. Myocardial fiber architecture in the human heart. Anatomical demonstration of modifications in the normal pattern of ventricular fiber architecture in a malformed adult specimen. Acta Anat 1990;138:352-8.

23 Matsuda H, Hirose $H$, Nakano S, Kishimoto H, Kato $H$, Kobayashi J, et al. Age-related changes in right and left Kobayashi J, et al. Age-related changes in right and left
ventricular function in tetralogy of Fallot. $尹$ pn Circ Res ventricular function

24 Toussaint M, Planche C, Duboc D, Pfister A, Da Lage C, Guerin F. Left ventricular ultrastructure in pulmonary stenosis and in tetralogy of Fallot. Virchows Archiv-A Pathol Histopathol 1987;411:33-8.

25 Alvarez L, Aranega A, Contreras JA, Lopez-Torres J, Fernandez JE. Morphometric study of right ventricle in 32 cases of tetralogy of Fallot. Herz 1988;13:41-8.

26 Jarmakami JM, Nakazawa M, Isabel-Jares J, Marks RA. Right ventricular function in children with tetralogy of Fallot before and after aortic-to-pulmonary shunt. Circulation 1976;53:555-61.

27 Lange PE, Onnasch DEW, Bernhard A, Heintzen PH. Left and right ventricular adaptation to right ventricular overCardiol 1982;50:786-94.

28 Gatzoulis MA, Clark AL, Cullen S, Newman CEH, Redington AN. Right ventricular diastolic function 15 to 35 years after repair of tetralogy of Fallot. Circulation 1995;91:1775-81.

29 Krymysky LD. Pathologic anatomy of congenital heart disease. Circulation 1965;32:814-27.

30 Weber KT, Sun Y, Tyagi SC, Cleutjens JPM. Collagen network of the myocardium: function, structural remod1994;26:279-92.

31 LeGrice IJ, Smaill BH, Chai LZ, Edgar SG, Gavin JB, Hunter PJ. Laminar structure of the heart: ventricular myocyte arrangement and connective tissue architecture in the dog. Am F Physiol 1995;38:H571-82.

32 Sanchez-Quintana D, Garcia-Martinez V, Climent V, Hurle JM. Morphological changes in the normal pattern of ventricular myoarchitecture in the developing human heart. Anat Rec 1995;243:483-95. 